\title{
Accuracy of multidetector computed tomography with maximum intensity projection technique in the assessment of fissure integrity
}

\author{
Stephan A. Soder ${ }^{1,2}$, Fabiola A. Perin ${ }^{2}$, José Carlos Felicetti ${ }^{2}$, José de Jesus P. Camargo ${ }^{2}$, \\ Spencer M. Camargo ${ }^{2}$, Bruno Hochhegger ${ }^{3}$, Paulo José Zimermann Teixeira ${ }^{4}$
}

${ }^{1}$ Health Sciences Postgraduation Program, Universidade Federal de Ciências da Saúde de Porto Alegre (UFCSPA), Porto Alegre, RS, Brazil; ${ }^{2}$ Division of Thoracic Surgery, Pavilhão Pereira Filho, Irmandade da Santa Casa de Misericórdia de Porto Alegre, Porto Alegre, RS, Brazil; ${ }^{3}$ Department of Radiology, University of Florida, Gainesville, FL, USA; ${ }^{4}$ Division of Pneumology, Pavilhão Pereira Filho, Irmandade da Santa Casa de Misericórdia de Porto Alegre, Porto Alegre, RS, Brazil

Contributions: (I) Conception and design: SA Soder, SM Camargo, B Hochhegger, PJZ Teixeira; (II) Administrative support: FA Perin, JC Felicetti, JJP Camargo, B Hochhegger, PJZ Teixeira; (III) Provision of study materials or patients: All authors; (IV) Collection and assembly of data: All authors; (V) Data analysis and interpretation: SA Soder, SM Camargo, B Hochhegger, PJZ Teixeira; (VI) Manuscript writing: All authors; (VII) Final approval of manuscript: All authors.

Correspondence to: Bruno Hochhegger, PhD. Department of Radiology, University of Florida, Gainesville, FL, USA.

Email: brunohochhegger@gmail.com.

Background: Bronchoscopic lung volume reduction (BLVR) is a potential treatment for patients with severe emphysema, performed through the placement of unidirectional endobronchial valves (EBVs). Their benefits are only achieved in patients that significantly reduce lobar volume, and it is mandatory that the fissures are complete. Fissure evaluation is preferably done by computed tomography, but little is known if its evaluation corresponds to the anatomical findings. The aim of this study is to evaluate the accuracy of thoracic radiologists in the identification of complete fissures by multidetector computed tomography (MDCT) using maximum intensity projection (MIP) technique, compared with direct anatomical evaluation. Methods: Prospective study, conducted in a single institution. Patients submitted to thoracic surgery had their fissures classified as complete or incomplete by thoracic surgeons and their preoperative chest scans evaluated by three radiologists, blinded for surgical evaluation. With the intraoperative categorization as a reference, the accuracy and concordance of the three thoracic radiologists' evaluation were calculated. The most experienced radiologist evaluated the fissures at two different moments to estimate the intra-observer agreement.

Results: There were included 67 patients, being 37 (55\%) males, with a mean age of 64 years. The accuracy of radiological identification of complete fissures ranged from $76.8 \%$ for left posterior oblique fissure to $85.1 \%$ for left anterior oblique fissure, with the best performance achieved by the most experienced radiologist. The concordance of the radiological evaluation for fissure integrity compared to the surgical assessment $(\mathrm{k})$ was $0.53-0.68$. Intra-observer agreement ranged from 0.74 to 0.87 .

Conclusions: The evaluation of the fissure integrity by MDCT analysis using MIP technique by thoracic radiologists had high accuracy among the thoracic radiologists.

Keywords: Emphysema; fissure integrity; multidetector computed tomography analysis (MDCT analysis)

Submitted Aug 17, 2021. Accepted for publication Dec 06, 2021.

doi: $10.21037 /$ jtd-21-1359

View this article at: https://dx.doi.org/10.21037/jtd-21-1359

$\wedge$ ORCID: 0000-0003-4771-6308.

(c) Journal of Thoracic Disease. All rights reserved. 


\section{Introduction}

Pulmonary emphysema was the third leading cause of death worldwide in 2019 (1). In patients with chronic dyspnea, poor exercise capacity and significant hyperinflation, lung volume reduction (LVR) can be considered in addition to the optimal medical management. The benefits with lung volume reduction surgery (LVRS) were shown in highly selected patients with severe emphysema predominantly in upper lobes and low exercise capacity (2), with durable responses (3). Progressive understanding of pathophysiology mechanisms and radiological and clinical characteristics of emphysema $(4,5)$ increased the enthusiasm to treat these patients through an endoscopic approach.

The unidirectional endobronchial valves (EBVs) are devices that only allow airflow out of lungs. They are inserted endoscopically in all segmental bronchi of target lobe to reduce lobar volume. The bronchoscopic lung volume reduction (BLVR) is indicated in patients with severe hyperinflation and heterogeneous emphysema, and can be considered to non-surgical candidates. Nevertheless, only the lobes with absent or minimal interlobar collateral ventilation $(\mathrm{CV})$ can effectively reduce their volume drastically, and the benefits of BLVR with EBV valves only can be achieved if significant lobar deflation occurs $(6,7)$.

$\mathrm{CV}$ is the ventilation between alveoli, bronchioles and even lobes by communications other than the main airway. Interlobar CV occurs mainly through the fissure. Physiologic evidence shows that interlobar CV gradually increases with age and becomes much more critical for lobar ventilation in emphysematous patients, primarily because of increased small airway pressure and higher resistance to airflow through bronchioles and bronchi $(8,9)$. In patients with significant air trapping for whom LVR is considered, the knowledge of fissure integrity is of paramount importance. If there are bridges of parenchyma connecting adjacent lobes, relevant $\mathrm{CV}$ is expected and the placement of EBV will be likely unsuccessful. On the other hand, selected patients with incomplete fissures and heterogeneous emphysema can be assessed for thoracoscopic LVR.

Target lobe volume reduction (TLVR) $>350 \mathrm{~mL}$ is considered to be significant and it has been used as a standard endpoint to define response after EBV placement. To identify patients who could reach TLVR $>350 \mathrm{~mL}$ after EBV insertion, both fissure integrity assessment through chest multidetector computed tomography (MDCT) analysis and $\mathrm{CV}$ estimative with Chartis system can be performed, with comparable accuracy, around 75-84\% (9-12). However, the agreement of MDCT fissure integrity analysis by radiologists with a reference standard, as the direct visualization in intra-operative of thoracic surgeries, has not been well described. Maximum intensity projection (MIP) is a data visualization method that enables viewing rays traced from the expected position of the operator through the object to the display screen, and only the relative maximum value detected along each ray path is retained by the computer. The MIP algorithm uses all the data in a volume of interest to generate a single bidimensional image. It is diagnostically useful because it can readily distinguish structures that are hyperdense with respect to surrounding tissues. In the fissure assessment, it may contribute to assess avascular perifissural area and highlight the fissure (13). The aim of this study is to evaluate the accuracy of thoracic radiologists to predict fissure completeness by reading MDCT scans and employing MIP technique, as compared to a reference standard being the intraoperative assessment by thoracic surgeons.

We present the following article in accordance with the STROBE reporting checklist (available at https://jtd. amegroups.com/article/view/10.21037/jtd-21-1359/rc).

\section{Methods}

\section{Ethical statement}

The study was conducted in accordance with the Declaration of Helsinki (as revised in 2013). The study was approved by Institutional Review Board of Santa Casa de Misericórdia de Porto Alegre (No. 2.387.846) (November $\left.20^{\text {th }}, 2017\right)$ and informed consent was waived from all the patients.

\section{Study design}

Prospective study and conducted at a single institution. Data collection was performed from December 2017 to May 2018. The patients included in the study had their fissures assessed intraoperatively during surgeries for treatment of pulmonary diseases. Likewise, patients had their MDCT scans analyzed anonymously and without interfering with patient care by the faculty or the attending hospital. The preoperative MDCT was evaluated by three radiologists dedicated to thoracic radiology, who were blinded to each other and to the intraoperative evaluation. 


\section{Patient selection}

Patients of 18 years or older who submitted to any lung resection surgery (video-assisted technique or thoracotomy), with preoperative high-resolution chest tomography (at a time interval shorter than or equal to 30 days to surgery) for assessment of their underlying disease, were selected.

For the purposes of this assessment, exclusionary criteria were CT scans performed before 30 days prior to surgery and/or who may have some confounding factor for the analysis of the fissure, such as the presence of pleural effusion, hilar or infiltrating fissural tumors, previous lung resection surgery or previous chemotherapy, radiotherapy or immunotherapy, and MDCT scans with poor resolution.

\section{Fissure graduation}

For completeness, the fissures were graded in two levels: (I) complete (when lobes were separated in more than $90 \%$ of its extension) and (II) incomplete (when the free portion was smaller or equal to $90 \%$ of the extension), according to a standard categorization (10). Both surgical evaluation and MDCT scans assessment used the same graduation to classify the fissure completeness.

For the purpose of grading, the right oblique (RO) fissure was divided in two halves, examined taking into account its posterior (superior) and anterior (inferior) halves, with the right horizontal $(\mathrm{RH})$ fissure as the landmark for this division; the left oblique (LO) was also divided in two portions for the evaluation, examined in its posterior (apical) and anterior (basal) halves, being pulmonary artery at the fissure the landmark for the division. The $\mathrm{RH}$ fissure was considered as a single portion because it didn't have the usual intersection of pulmonary artery and is smaller in length. Therefore, five fissure portions were evaluated: right oblique posterior (ROP), right oblique anterior (ROA), $\mathrm{RH}$, left oblique posterior (LOP) and left oblique anterior (LOA).

Location of the fissure defect may have clinical implication, especially for the right upper lobe, which is delineated by the RH and the superior half of the RO. In case this lobe is selected as target for EBV therapy, these are the fissure components most important to be addressed.

\section{Intraoperative assessment}

Only the fissures on the operated side were evaluated. Intraoperative (surgical) analysis was performed after opening pleural cavity, prior to any dissection of hilar structures. Fissure analysis was performed without implying any additional risk to the patient in the surgical procedure, since the evaluation of pleural cavity and pulmonary fissures in resections is routinely performed. The fissure was considered complete when the pulmonary artery was visible in the fissure with minimal amount of tissue covering its adventitia and the parenchyma in the length between the costal (lateral) and mediastinal surfaces was scarce or absent ( $>90 \%$ integrity). When these criteria were not satisfied, the fissure was classified as incomplete ( $\leq 90 \%$ integrity) (14).

All patients had their pulmonary fissures evaluated by two thoracic surgeons (SS, 3 years of experience; FP, 16 years of experience; SC, 21 years of experience; JF, 42 years of experience; JC, 45 years of experience) at the beginning of the surgery. When both agreed with the fissure completeness, the consensus was reached. In the case of disagreement, a third surgeon was called to evaluate the fissure to reach the consensus. The intraoperative consensus was considered the standard reference for the graduation of the fissure completeness.

\section{MDCT scans}

All volumetric data were obtained using a 64-detector (LightSpeed VCT 64 slice scanner GE Healthcare, Waukesha, WI, USA). Scans were obtained at endinspiration breath-hold, without intravenous administration of iodinated contrast media. CT parameters were: $120-\mathrm{kV}$ tube voltage, $210 \mathrm{mAs}$ (range, 110-270 mAs) tube current and $0.625 \mathrm{~mm}$ slice thickness. Image data was reconstructed using standard and high-resolution algorithm, $1.0 \mathrm{~mm}$ reconstruction interval, field of view of $350-400 \mathrm{~mm}$ and a $512 \times 512$ matrix. All datasets were transferred to a PACS system (Synapse, Fuji Medical System, Lexington, MA, USA) at calibrated workstations.

\section{Imaging evaluation}

Three chest radiologists (BH, 14 years of experience; MCB, 10 years of experience; GTA, 7 years of experience) retrospectively reviewed all MDCT images separately, blinded to any clinical information, using the GE Advantages Windows workstation (GE Medical System, Milwaukee, WI, USA). In addition, the most experienced thoracic radiologist evaluated all the images with a time difference of 90 days, in order to evaluate the intra-observer agreement of the fissure evaluation by MDCT scan.

Readers were instructed to review the images as they 
would do in their regular daily practice. Any user-defined reconstructions, were available for review including axial images and multiplanar reformatted (MPR) images. Dynamic image presentation with "scrolling through" the data sets was available to facilitate the detection of structures. There were no time constraints for image interpretation. The readers had previous experience with fissure evaluation and also used the interactive multiplanar reformation tools to help categorizing each fissure. All CT scans were reformatted with MIP technique with $8 \mathrm{~mm}$ of thickness in sagittal and coronal planes (15). The pulmonary fissures were analyzed using the standard lung window settings (level: $-600 \mathrm{HU}$, width: 1,600 HU). The definition of CT fissure was hyper attenuation line in avascular topography of anatomical pulmonary fissure. The images were used for the purpose of the semi-quantitative analysis, and fissure integrity was estimated in $>90 \%$ integrity (complete) and $\leq 90 \%$ integrity (incomplete). The RO fissure was examined taking into account its superior and inferior halves, with the $\mathrm{RH}$ fissure as the landmark for this division; the LO was examined in its apical and basal halves. Location of the fissure defect may have clinical implication, especially for the right upper lobe, which is delimited by the RH and the superior half of the RO. In case this lobe is selected as target for EBV therapy, these are the fissure components most important to be addressed. In this study, we used such subdivisions to identify if the readers were describing the same fissural defects. A fissure was considered fully complete only if it could be identified in all its extension, with special attention to the medial attachment at the mediastinum and hilum.

\section{Outcomes}

To evaluate the accuracy of three dedicated thoracic radiologists to identify a complete fissure by MDCT scan analysis, compared to a standard reference being the fissural assessment by thoracic surgeons in intraoperative time. Additionally, we measured the accuracy of fissure analysis by the same radiologist in distinct moments, 90 days separated.

\section{Statistical analysis}

Data were presented as frequency and percentage. We used the Kappa coefficients to assess accuracy of radiological evaluation and intra-observer agreement. The interpretation was conducted based on the following parameters: Kappa of
0.01 indicates "very weak" agreement; Kappa from 0.01 to 0.20 indicate "weak" agreement; Kappa from 0.21 to 0.40 indicate "low" agreement; Kappa from 0.41 to 0.60 indicates "moderate" agreement; Kappa of 0.61 to 0.80 indicate "substantial" agreement; Kappa from 0.81 to 1.00 indicate "almost perfect" agreement (16). As a reference, we used the evaluation of the fissures by the surgeon to calculate sensitivity, specificity, positive and negative predictive values and accuracy of radiologist evaluation in correctly identify complete fissures. In all cases, $\mathrm{P}$ values of less than 0.05 were considered statistically significant. Data analysis was performed through the SPSS v.22 program (IBM, Chicago, IL, USA).

\section{Results}

Sixty-seven patients met the inclusion and exclusion criteria. Thirty-seven $(55 \%)$ were male, and the mean age was 64 years old. Forty-four $(66 \%)$ procedures were on the right side and $23(34 \%)$ on the left side. A total of 178 fissure portions was assessed. The proportion of emphysema patients was $45 \%$. The highest prevalence of complete fissures was of the LOA portion $(61 \%)$ and the lowest was of the RH fissure (26\%) (Figures 1,2). Clinical and demographic characteristics are shown in Table 1.

The mean accuracy of the prediction of complete fissures through the evaluation of MDCT scans by radiologists varied between $76.81 \%$ for the left posterior oblique portion and $85.06 \%$ for the left anterior oblique portion. The mean accuracy for RH fissure, usually the most frequently incomplete and most difficult to define precisely, was $80.62 \%$, varying slightly between the three radiologists, from $74.42 \%$ (radiologist C) to $83.72 \%$ (radiologists A and B). Positive predictive values were higher for LOA fissure (94.45\%) and lower for RH fissure (70.83\%). Radiologist A, the most experienced, achieved a higher accuracy among radiologists. Sensitivity, specificity, positive and negative predictive values, and accuracies are shown in Table 2 and Figure 1. We demonstrate two examples of fissures visualized in the surgery and in the MDCT (Figures 2,3).

The agreement of MDCT fissure integrity of radiologists compared to intraoperative evaluation varying between 0.53 (ROA fissure) and 0.68 (LOA fissure). The most experienced radiologist (radiologist A) achieved a higher agreement $(\mathrm{k}=0.60-0.82)$, as expressed in Table 3. The intraobserver agreement of radiologist A was very good $(\mathrm{k}=0.74$ 0.87), as shown in Table 4. 


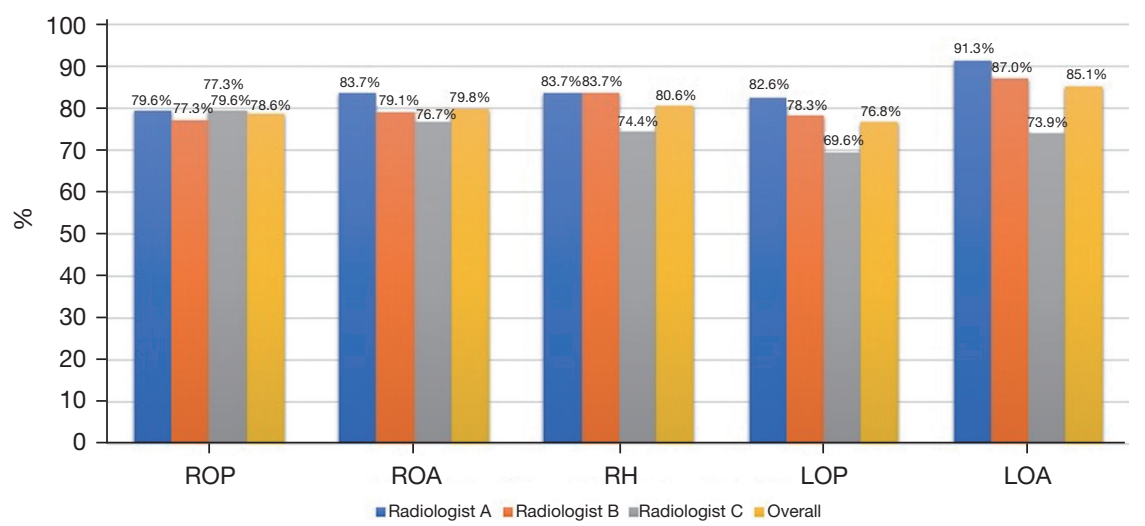

Figure 1 Accuracy of thoracic radiologists' assessment of fissure integrity accordingly to the fissure. ROP, right oblique posterior; ROA, right oblique anterior; RH, right horizontal; LOP, left oblique posterior; LOA, left oblique anterior.
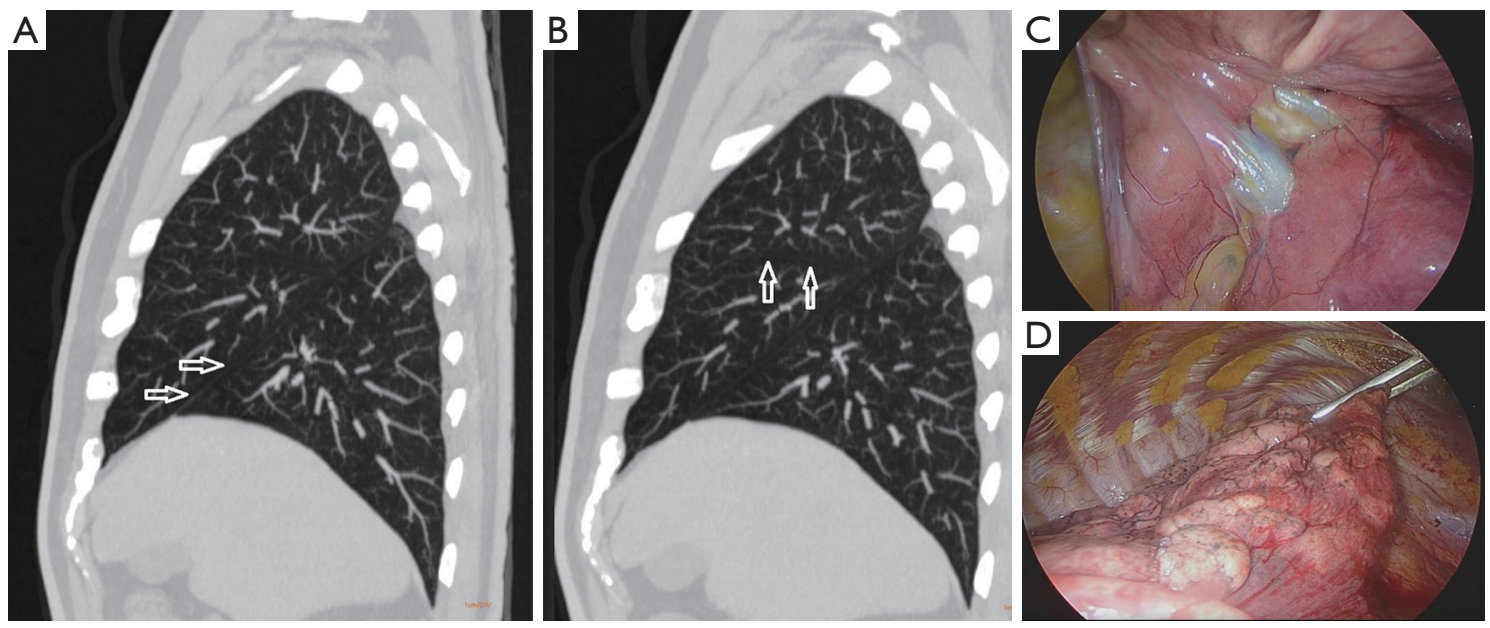

Figure 2 Fifty-six yo male, undergoing lung resection for lung nodule. In CT sagittal images with MIP technique showing complete oblique fissure (arrows in A) and incomplete horizontal fissure (arrows in B); surgical correlations confirm oblique fissure integrity (C) and incomplete horizontal fissure (D). MIP, maximum intensity projection.

\section{Discussion}

LVR strategies are useful treatments for selected hyperinflated and poor performance emphysematous patients. The largest trial to evaluate the impact of LVRS, the National Emphysema Treatment Trial (NETT), identified a group who responded most favorably to LVRS: those with upper lobe-predominant emphysema with low baseline exercise capacity (2). Better understanding of clinical and physiologic features of emphysema, development of minimally invasive techniques and the constant improvement of anesthetic and postoperative care have contributed to reach the benefits of LVR with lower morbidity and mortality. Among several clinical, functional and tomographic parameters evaluated in these patients, the understanding of the fissure integrity plays a fundamental role in the decisionmaking. Fissure completeness is analyzed as a surrogate for interlobar collaterals. If a target lobe is identified for BLVR, the fissure must be complete so that no significant interlobar $\mathrm{CV}$ occurs, and deflation can be achieved. When the fissure is incomplete, treatment with EBV will not be successful and the patient could be considered for thoracoscopic LVRS.

Several studies have compared the prediction capacity to identify responders after EBV placement, by means of achieving TLVR $>350 \mathrm{cc}$, between the Chartis and fissure 
Table 1 Patient's clinical and demographic characteristics

\begin{tabular}{|c|c|}
\hline Parameter & $\mathrm{N}=67$ \\
\hline Male & $37[55]$ \\
\hline Age, years & $64 \pm 14$ \\
\hline $\mathrm{BMI}, \mathrm{kg} / \mathrm{m}^{2}$ & $25.6 \pm 4.5$ \\
\hline \multicolumn{2}{|l|}{ Smoking status } \\
\hline Never smoker & $18[27]$ \\
\hline Current smoker & 15 [22] \\
\hline Past smoker & 34 [51] \\
\hline Pack years smoking & $49 \pm 33$ \\
\hline $\mathrm{FEV}_{1}, \%$ of predicted & $78 \pm 20$ \\
\hline \multicolumn{2}{|l|}{ Diagnosis } \\
\hline Adenocarcinoma & $32[48]$ \\
\hline Epidermoid or squamous cell carcinoma & 14 [21] \\
\hline Neuroendocrine tumors & $7[10]$ \\
\hline Benign diseases & 14 [21] \\
\hline \multicolumn{2}{|l|}{ Surgery } \\
\hline Lobectomy & $44[66]$ \\
\hline Wedge resection & 6 [9] \\
\hline Other & 17 [25] \\
\hline \multicolumn{2}{|l|}{ GOLD classification } \\
\hline No emphysema & $37[55]$ \\
\hline I & $5[7]$ \\
\hline II & 22 [33] \\
\hline III & $3[5]$ \\
\hline \multicolumn{2}{|l|}{ Complete fissure $^{a}$} \\
\hline Right oblique posterior & 19 [43] \\
\hline Right oblique anterior & 27 [61] \\
\hline Right horizontal & $11[26]$ \\
\hline Left oblique posterior & 9 [39] \\
\hline Left oblique anterior & $12[52]$ \\
\hline
\end{tabular}

Data were presented as No. (\%) or mean \pm SD. ${ }^{\text {a }}$, assessed by thoracic surgeon. BMI, body mass index; $\mathrm{FEV}_{1}$, forced expiratory volume in first second; GOLD, global initiative for chronic obstructive lung disease.

integrity analysis by MDCT visual assessment performed by a radiologist. The performance of both methods is often reported to be equivalent in this regard, with accuracy ranging from $74 \%$ to $83 \%$ (10-12), and potentially increased when both methods are combined, with reported accuracy of $89 \%$ (12).

The MIP technique is based on the principle of keeping the object with the highest density per slice only and reformatting slices thicker than original slice thickness (17). Because of the better traceability of intrapulmonary vascular structures, this technique makes nodules or fissure adjacent to them easier to percept. With the progressive improvement of imaging quality, potential movement artifacts which may affect fissure integrity interpretation can be reduced, as well small fissure gaps can be better assessed in how significant they will be for $\mathrm{CV}$ in severe emphysema patients. The use of this technique might explain our better results than previously reported $(15,18)$. However, no direct comparison of the performance between radiologic evaluation using and not using MIP technique was performed, therefore limiting conclusions regarding the specific benefit of adding this algorithm to the routine evaluation of the fissure completeness. The addition of quantitative analysis of fissure integrity by mathematic algorithms can also improve the prediction of patients with CV absent (19).

The fissure evaluation by MDCT scans is considered to be quite accurate, but little is known if these findings closely correspond to the anatomical categorization by direct inspection. In our study, we found a very good accuracy of all radiologists in the fissure assessment compared to the intra-operative findings in lung surgeries. Overall accuracy rates around $80 \%$ and reaching $85 \%$ for LOA portion are important for daily clinical decisions. Even for RH fissure, considered the most difficult to assume as complete and with lower accuracy of MDCT scans analysis (16), we found a high accuracy $(80.6 \%)$ of this evaluation. Sensitivity was higher for RO fissures (85\% for ROP and $95 \%$ for ROA fissure), and the positive predictive value was higher for LOA fissure (94\%). The division of oblique fissures in two halves is critical, for example, for the right side, considering that upper lobe is bordered by posterior half of oblique fissure and horizontal fissure. Thus, although the anterior half is incomplete, this will not affect the CV of the upper lobe.

Agreement between radiologists evaluating fissure integrity is variable in the literature. Koenigkam-Santos et al. (15) found $\mathrm{k}=0.67-0.76$, and Guan et al. (18) detected a $\mathrm{k}=0.59-0.65$. In this study, the agreement between radiologists as having the surgical evaluation as the reference was moderate to high, and it was comparable to previously reported studies. The better performance of the 
Table 2 Diagnostic capability of thoracic radiologists' assessment of fissure integrity compared to direct visualization in intra-operative of thoracic surgeries

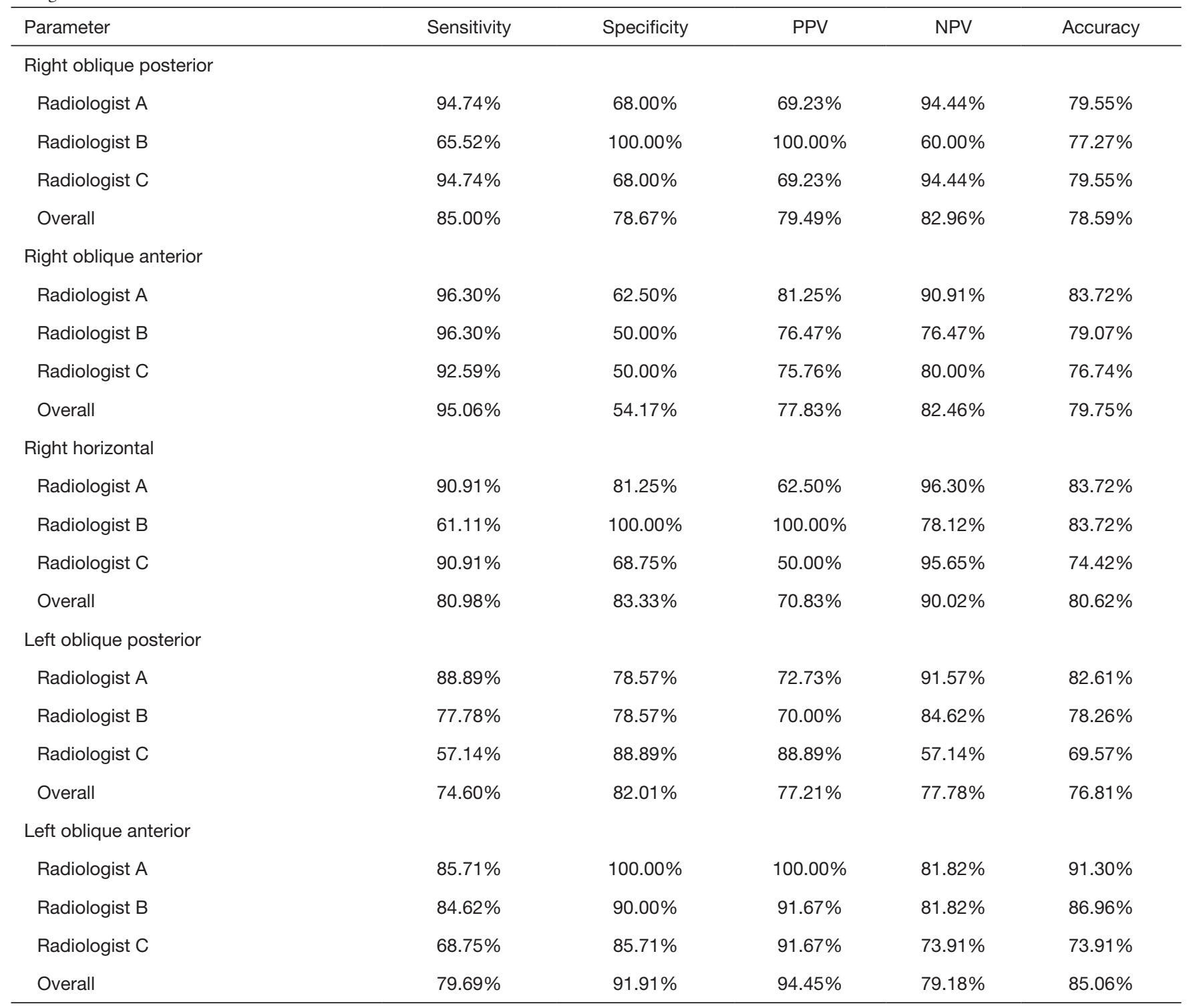

PPV, predictive positive value; NPV, negative predictive value.

most experienced radiologist reinforces the importance of training and continuous education, allowing for more precise classification of fissure completeness and, therefore, improved selection of patients for potential LVR therapies. The very good intra-observer agreement in this study $(\mathrm{k}=0.74-0.87)$ reflects that their conclusions are little affected by time or chance.

As a limitation of our study, the majority of patients had no emphysema (55\%). It is known that $\mathrm{CV}$ is greater in emphysematous patients and the existence of fissural gaps may be more relevant for $\mathrm{CV}$ in this population. Further, the anatomic modifications and heterogeneity of severely damaged lung by emphysema make the fissure assessment considerably more difficult (15). However, the prevalence of complete fissures is considered to be similar between the different degrees of emphysema (20). As candidate patients to BLVR are usually not operated, it is very difficult to find Global Initiative for Chronic Obstructive Lung Disease (GOLD) grade three or four patients that undergone lung surgery to evaluate fissures and be the reference standard in 

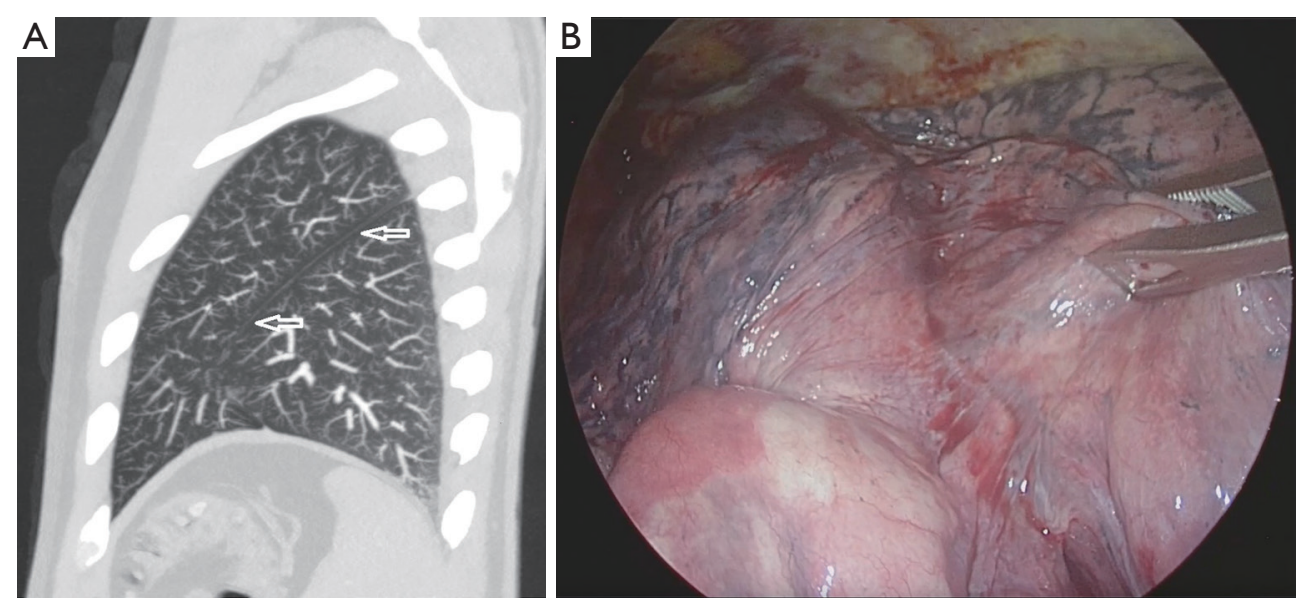

Figure 3 Thirty-eight yo male, undergoing VATS left upper lobectomy. In CT sagittal images with MIP technique showing incomplete oblique fissure (arrows in A); surgical correlations confirm incomplete oblique fissure (B). VATS, video-assisted thoracoscopic surgery; MIP, maximum intensity projection.

Table 3 Concordance of fissure integrity between thoracic radiologists to direct visualization in intra-operative of thoracic surgeons

\begin{tabular}{llllll}
\hline & \multicolumn{3}{c}{ Thoracic surgeons } \\
\cline { 2 - 6 } Kappa & ROP & ROA & RH & LOP & 0.65 \\
\hline Radiologist A & 0.60 & 0.63 & 0.62 & 0.82 \\
Radiologist B & 0.56 & 0.51 & 0.64 & 0.55 & 0.74 \\
Radiologist C & 0.60 & 0.46 & 0.47 & 0.42 & 0.54 \\
Overall & 0.59 & 0.53 & 0.58 & 0.68 & 0.47 \\
\hline
\end{tabular}

ROP, right oblique posterior; ROA, right oblique anterior; $\mathrm{RH}$, right horizontal; LOP, left oblique posterior; LOA, left oblique anterior.

Table 4 Intra-observer concordance of fissure integrity of thoracic radiologist

\begin{tabular}{llllll}
\hline \multirow{2}{*}{ Kappa } & \multicolumn{4}{c}{ Radiologist A, time zero } \\
\cline { 2 - 6 } & ROP & ROA & RH & LOP & LOA \\
\hline Radiologist A, 90 days after & 0.81 & 0.87 & 0.76 & 0.74 & 0.81 \\
\hline
\end{tabular}

$\mathrm{ROP}$, right oblique posterior; ROA, right oblique anterior; $\mathrm{RH}$, right horizontal; LOP, left oblique posterior; LOA, left oblique anterior.

a similar study.

In this study, we observe that the accuracy of the MDCT scans analysis using MIP technique in identifying complete fissures, in a population predominantly non-emphysematous or GOLD I and II, is similar to its prediction capacity of identifying responders (TLVR $>350 \mathrm{~mL}$ ) after EBV insertion in other studies (12), whose population has more severe degrees of emphysema. Thus, despite of potential differences in radiological presentations, the ability to identify complete fissures through MDCT scans may not be very different between the different degrees of emphysema and these results may be extrapolated to GOLD three or four patients. The higher availability and feasibility of MDCT scans to daily practice, associated with high accuracy rates to identify complete fissures in emphysematous patients make this method a useful tool for selecting candidates for the appropriate intervention alternatives for LVR. 


\section{Conclusions}

Our results show that fissure integrity evaluation through MDCT scan analysis using MIP technique for trained radiologists have a very good accuracy when compared to the intra-operative evaluation of lung fissures. A trustable interpretation of this parameter has large clinical impact and it is helpful in order to select candidates with complete fissures for EBV placement and those with incomplete fissures for potential thoracoscopic approach.

\section{Acknowledgments}

Funding: None.

\section{Footnote}

Reporting Checklist: The authors have completed the STROBE reporting checklist. Available at https://jtd. amegroups.com/article/view/10.21037/jtd-21-1359/rc

Data Sharing Statement: Available at https://jtd.amegroups. com/article/view/10.21037/jtd-21-1359/dss

Peer Review File: Available at https://jtd.amegroups.com/ article/view/10.21037/jtd-21-1359/prf

Conflicts of Interest: All authors have completed the ICMJE uniform disclosure form (available at https://jtd.amegroups. com/article/view/10.21037/jtd-21-1359/coif). The authors have no conflicts of interest to declare.

Ethical Statement: The authors are accountable for all aspects of the work in ensuring that questions related to the accuracy or integrity of any part of the work are appropriately investigated and resolved. The study was conducted in accordance with the Declaration of Helsinki (as revised in 2013). The study was approved by Institutional Review Board of Santa Casa de Misericórdia de Porto Alegre (No. 2.387.846) (November 20th, 2017) and informed consent was waived from all the patients.

Open Access Statement: This is an Open Access article distributed in accordance with the Creative Commons Attribution-NonCommercial-NoDerivs 4.0 International License (CC BY-NC-ND 4.0), which permits the noncommercial replication and distribution of the article with the strict proviso that no changes or edits are made and the original work is properly cited (including links to both the formal publication through the relevant DOI and the license). See: https://creativecommons.org/licenses/by-nc-nd/4.0/.

\section{References}

1. World Health Organization. Chronic obstructive pulmonary disease (COPD) [Internet]. 2021. Available online: https://www.who.int/en/news-room/fact-sheets/ detail/chronic-obstructive-pulmonary-disease-(copd)

2. Fishman A, Martinez F, Naunheim K, et al. A randomized trial comparing lung-volume-reduction surgery with medical therapy for severe emphysema. N Engl J Med 2003;348:2059-73.

3. Yusen RD, Lefrak SS, Gierada DS, et al. A prospective evaluation of lung volume reduction surgery in 200 consecutive patients. Chest 2003;123:1026-37.

4. Criner GJ, Cordova F, Sternberg AL, et al. The national emphysema treatment trial (NETT): Part I: Lessons learned about emphysema. Am J Respir Crit Care Med 2011;184:763-70.

5. Criner GJ, Cordova F, Sternberg AL, et al. The national emphysema treatment trial (NETT) Part II: Lessons learned about lung volume reduction surgery. Am J Respir Crit Care Med 2011;184:881-93.

6. Sciurba FC, Ernst A, Herth FJ, et al. A randomized study of endobronchial valves for advanced emphysema. $\mathrm{N}$ Engl J Med 2010;363:1233-44.

7. Davey C, Zoumot Z, Jordan S, et al. Bronchoscopic lung volume reduction with endobronchial valves for patients with heterogeneous emphysema and intact interlobar fissures (the BeLieVeR-HIFi study): a randomised controlled trial. Lancet 2015;386:1066-73.

8. Higuchi T, Reed A, Oto T, et al. Relation of interlobar collaterals to radiological heterogeneity in severe emphysema. Thorax 2006;61:409-13.

9. Koster TD, Slebos DJ. The fissure: interlobar collateral ventilation and implications for endoscopic therapy in emphysema. Int J Chron Obstruct Pulmon Dis 2016;11:765-73.

10. Gompelmann D, Eberhardt R, Slebos DJ, et al. Diagnostic performance comparison of the Chartis System and highresolution computerized tomography fissure analysis for planning endoscopic lung volume reduction. Respirology 2014;19:524-30.

11. Schuhmann M, Raffy P, Yin Y, et al. Computed tomography predictors of response to endobronchial valve lung reduction treatment. Comparison with Chartis. Am J 
Respir Crit Care Med 2015;191:767-74.

12. Koster TD, van Rikxoort EM, Huebner RH, et al. Predicting lung volume reduction after endobronchial valve therapy is maximized using a combination of diagnostic tools. Respiration 2016;92:150-7.

13. Perandini S, Faccioli N, Zaccarella A, et al. The diagnostic contribution of CT volumetric rendering techniques in routine practice. Indian J Radiol Imaging 2010;20:92-7.

14. Kent MS, Ridge C, O'Dell D, et al. The accuracy of computed tomography to predict completeness of pulmonary fissures. A prospective study. Ann Am Thorac Soc 2015;12:696-700.

15. Koenigkam-Santos M, Puderbach M, Gompelmann D, et al. Incomplete fissures in severe emphysematous patients evaluated with MDCT: incidence and interobserver agreement among radiologists and pneumologists. Eur J Radiol 2012;81:4161-6.

16. Landis JR, Koch GG. The measurement of observer

Cite this article as: Soder SA, Perin FA, Felicetti JC, Camargo JJP, Camargo SM, Hochhegger B, Teixeira PJZ. Accuracy of multidetector computed tomography with maximum intensity projection technique in the assessment of fissure integrity. $\mathrm{J}$ Thorac Dis 2022;14(1):54-63. doi: 10.21037/jtd-21-1359 agreement for categorical data. Biometrics 1977;33:159-74.

17. Li WJ, Chu ZG, Zhang Y, et al. Effect of Slab Thickness on the Detection of Pulmonary Nodules by Use of CT Maximum and Minimum Intensity Projection. AJR Am J Roentgenol 2019;213:562-7.

18. Guan CS, Xu Y, Han D, et al. Volumetric thin-section CT: evaluation of pulmonaryinterlobar fissures. Diagn Interv Radiol 2015;21:466-70.

19. Fiorelli A, Poggi C, Anile M, et al. Visual analysis versus quantitative CT analysis of interlobar fissure integrity in selecting emphysematous patients for endobronchial valve treatment. Interact Cardiovasc Thorac Surg 2019;28:751-9.

20. Koenigkam-Santos M, de Paula WD, Owsijewitsch M, et al. Incomplete pulmonary fissures evaluated by volumetric thin-section CT: semi-quantitative evaluation for small fissure gaps identification, description of prevalence and severity of fissural defects. Eur J Radiol 2013;82:2365-70. 